\title{
Energy storage systems for wind power application
}

\author{
Raúl Sarrias $^{1}$, Luis M. Fernández ${ }^{1}$, Carlos A. García ${ }^{1}$, and Francisco Jurado ${ }^{2}$ \\ ${ }^{1}$ Department of Electrical Engineering, University of Cadiz, EPS Algeciras, \\ Avda. Ramón Puyol, s/n. 11202 Algeciras (Cádiz), Spain \\ e-mail: raul.sarriasmena@alum.uca.es, luis.fernandez@uca.es, carlosandres.garcia@uca.es \\ 2 Department of Electrical Engineering, University of Jaen, EPS Linares, \\ C/ Alfonso X, $\mathrm{n}^{\circ}$ 28. 23700 Linares (Jaén), Spain \\ e-mail: fjurado@ujaen.es
}

\begin{abstract}
Since energy consumption and pollution levels are increasing worldwide, the traditional fossil fuel-based generation methods are open to debate. A considerable number of countries are promoting the use of renewable resources in order to set a new energetic scenario.
\end{abstract}

Wind power generation needs to improve some specific aspects that hinder its development. Several devices have been designed and are currently in use to solve the problem of energy volatility, since they are able to store energy in different forms for a given period of time. All these devices have different characteristics; therefore, they behave dissimilarly under certain operating conditions.

Lead-acid batteries are being used either for energy or power applications. However, there exist other devices that may provide better performance. These devices are here described and compared, coming to the conclusion that some of them will substitute current technology in the near future if they are able to improve several indicated aspects.

\section{Key words}

Wind power, battery, supercapacitor, flywheel, superconducting magnetic energy storage, hydrogen energy.

\section{Introduction}

In all the industrialised countries, an efficient energy supply is basic for a proper economic enhancement. Energy is present in different forms in every daily activity. Traditionally, the way in which this energy was produced, transported and consumed was not an aspect to worry about. Resources were enough for many decades and pollution did not concern most of the population. However, an attempt to find less environmentally hazardous means of supplying energy is being made nowadays. Here is where renewable energies appear as a cleaner alternative, being wind power generation one of the most extended options. Nevertheless, in order to develop new electric systems with larger participation of wind power, it is necessary to overcome some of the drawbacks it presents.

In this study, the storage problem is dealt. Important researches are being carried out with the aim of improving the already existing, and developing new techniques that will allow more effective storage systems. Several internationally acknowledged references have been revised to elaborate this paper.

In this work, the electrochemical battery, redox flow battery, sodium sulphur battery, flywheel, supercapacitor, SMES system and the hydrogen technology are studied, since they are considered some of the most interesting alternatives. All these technologies are described by means of their main working principles. In addition, a comparison of the stated technologies is done attending to the most remarkable capabilities required to operate properly under given conditions. The conclusions drawn from the previous analysis are articulated in order to provide some ideas of how these technologies ought to evolve.

\section{Energy Storage Technologies}

In this section, the different storage technologies utilised nowadays will be analysed in depth. For each of them, its working principles and most remarkable characteristics will be described.

\section{A. Electrochemical Batteries}

Different types of batteries are considered in this paper. This section includes the electrochemical batteries most widely used, as well as those whose second-rate importance do not require a deeper study.

Electrochemical accumulators are divided into several cells, formed by electrodes, electrolyte and electrical insulator, where electrochemical reactions occur. When supplying energy to a battery, its internal compounds react, allowing energy storage in a chemical form. This energy is released as electricity when a load is connected 
to the battery. Therefore, reversible transformations take place, charging and discharging the battery respectively, by electrons transference. Voltage and current outputs are fixed by the electrochemical reactions developed. However, these features can adapt to requirements by interconnecting, in series or parallel, multiple batteries modules.

Electrochemical batteries can be divided into three major groups [1]: Lead-acid, which is currently the leading technology; lithium-based, with important applications in the field of portable devices; and nickel-based.

Lead-acid batteries consist of a lead dioxide positive electrode and a sponge lead negative electrode, both immersed in a sulphuric acid solution which works as electrolyte, with a micro-porous material for electrical insulation [2]. Among lithium-based batteries, the Li-ion type is currently the most interesting one, with large appliance in portable devices, above Li-polymer, which shows a shorter lifetime. Lithium-ion batteries comprise a lithium-metal oxide and graphitic carbon as positive and negative electrodes respectively, using lithium salts for the electrolyte. In all the existing nickel-based batteries, the positive electrode is made of nickel hydroxide, whereas an aqueous solution of potassium hydroxide and lithium hydroxide is used as electrolyte [1]; with a cadmium hydroxide negative electrode in the most frequently used model.

Eventually, a developing technology, metal-air batteries, uses aluminium or zinc for negative electrodes, as for the positive (or air) electrodes, porous carbon structures are utilised. The electrolyte can be either a liquid or a solid membrane with potassium hydroxide [2].

\section{B. Redox Flow Battery}

Also called simply flow batteries, this technology follows the same principles as the previously described with a different working technique. The system uses two electrolytes, which are stored in separated tanks. When necessary, both electrolytes are pumped to a cell, where the electrodes are separated by an ion-selective membrane, thus allowing the charge or discharge of the battery via ion exchange between the electrolytes (Fig. 1). This technology allows decoupling the energy and power capacities, since available energy depends on the volume of electrolytes stored in the reservoir tanks, whereas the power rates are limited by the ions transfer speed in the cell.

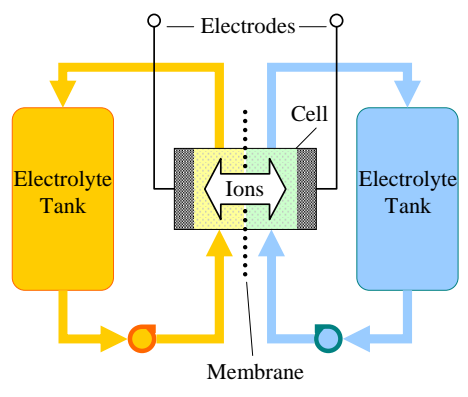

Fig. 1 - Redox Flow Battery Scheme

\section{Sodium Sulphur Battery (NaS)}

Positive and negative electrodes are comprised of molten sulphur and molten sodium respectively. Between them, a solid beta alumina ceramic electrolyte is placed (Fig. 2). This electrolyte allows ion exchange for the electrochemical reactions to happen. To maintain sulphur and sodium in liquid state, a high temperature is needed, typically from 300 to $350^{\circ} \mathrm{C}$. Therefore, heat must be provided for the reaction to start; however, once it is running, the heat produced by continuous charging and discharging cycles is enough to maintain temperature in the required range [1], thus avoiding the need for an external heat supply.

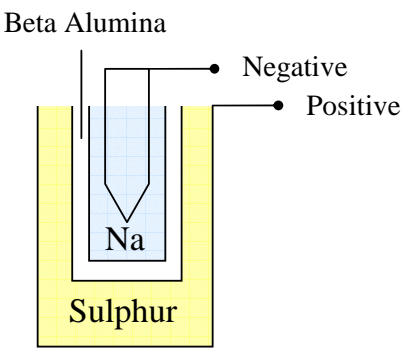

Fig. 2 - NaS Battery Scheme

\section{Flywheel}

This device consists of a mechanical system that stores kinetic energy in a rotating mass. The stored energy is proportional to the rotor speed and its moment of inertia; the higher these parameters are the more rotational energy it accumulates. Therefore, two options appear. Low-speed flywheels use heavy steel rotor with high inertia. On the other hand, high-speed flywheels use composite materials in order to obtain a lighter rotor. In some cases, magnetic bearings and low pressure vessels are utilised with the aim of reducing internal losses and self-discharge (Fig. 3).

The system also presents a reversible motor/generator, which is responsible for accelerating the rotating mass when storing kinetic energy and decelerating when obtaining electricity from the rotor. This device is the linkage between the electric machinery and the rotating system. Both the rotor and the motor/generator are coupled to the same shaft.

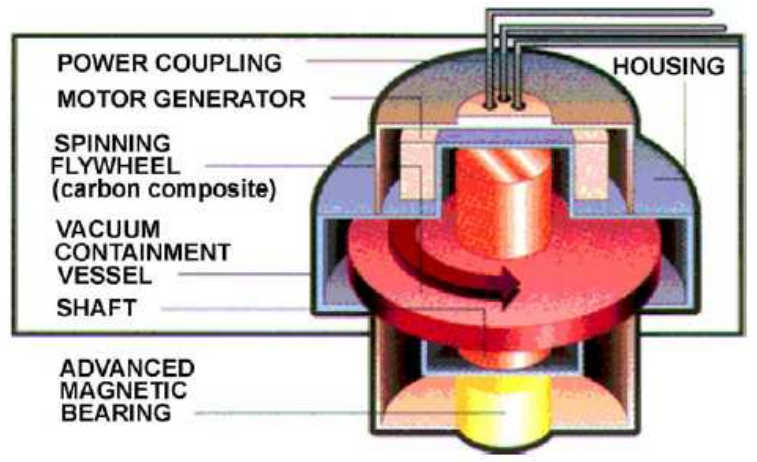

Fig. 3 - Flywheel Scheme [1] 


\section{E. Supercapacitor}

A supercapacitor is based on the same principles as a common capacitor. However, using a specially designed structure, it allows a much higher capacitance per unit volume. Energy is stored by action of the electric field existing between two electrodes separated, only a few $\AA$ distance, by a liquid electrolyte. As a consequence, since no chemical reaction occurs, its cycling capacity exceeds that of the batteries, thus having a longer lifespan when operating under continuous and deep charge/discharge cycles.

The most commonly used type is the Electrochemical Double Layer Capacitor (EDLC). The EDLC consists of two electrodes, which are normally made of porous active carbon, carbon aerogels or carbon nanotubes in the most recently developed technologies [1], whereas the electrolyte can be aqueous or organic. This structure provides an exceptional surface-mass rate. The voltage output depends on the characteristics of the model utilised. Nevertheless, supercapacitors can be arranged in order to reach specific requirements.

\section{F. Superconducting Magnetic Energy Storage (SMES)}

This technology requires complex systems that store energy in strong magnetic fields created by the flow of direct current through a superconducting coil. The superconducting materials of the coil require very low temperatures. Hence, the system is divided into two main blocks: the cold components and the cooling system. The cold components are immersed in liquid helium -at about $-270^{\circ} \mathrm{C}-$ in a vacuum enclosure. The cooling system is responsible for cooling and pumping liquid helium to the enclosure. This operation consumes relatively large amounts of energy, what reduces economic competitiveness against other technologies.

Since DC is provided to generate magnetic fields, an $\mathrm{AC} / \mathrm{DC}$ reversible converter is needed in order to manage current input and output when the system is connected to the electricity network.

\section{G. Hydrogen}

The hydrogen technology comprises three different stages, which can be developed in varied locations. The first stage involves hydrogen production. This procedure takes place in an electrolyzer, where water splits in hydrogen and oxygen. This chemical reaction requires energy supply, and, as a result, oxygen is normally released to the atmosphere whereas hydrogen is stored.

Hydrogen storage is a complex operation. A number of investigations are being carried out in order to improve the already existing technologies, mainly by reducing hydrogen losses from the tanks. To sum it up, four main technologies are currently available [1]; hydrogen pressurisation and adsorption in metal hydrides are more developed, while liquefaction and adsorption on carbon nanofibres are still under research.
Finally, stored hydrogen reacts with oxygen in a fuel cell. Via inverse electrolysis, energy is obtained and water is wasted without generating hazardous effects on the environment. Fuel cells consist of a two electrodeselectrolyte structure. Depending on the materials used, different types of fuel cells appear.

As seen in Fig. 4, a combined wind-hydrogen system can operate either connected to grid or isolated. Furthermore, hydrogen can be stored and consumed in a fuel cell, developing the complete energetic cycle in the wind farm; or otherwise, transported to a different location and used in an external system.

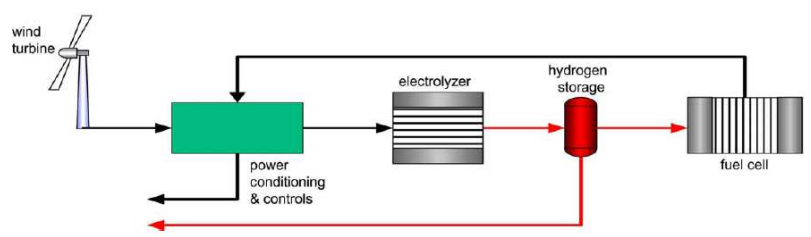

Fig. 4 - Combined Wind-Hydrogen Scheme [3]

\section{Working Features}

The previously presented storage devices are able to execute different functions in the electricity grid. Therefore, one particular device will be chosen or rejected according to the specific requirements of each application, since they all have different working features.

It is always possible to classify the applications of largescale energy storage devices into one of the following:

- Power Quality: In this application, the grid is supplied with stored energy for a few seconds or less. The aim is to ensure the proper quality of the electric energy provided to the consumers. This is a very common performance when using the storage with wind power, since it allows reducing the short-term fluctuations produced by the variability of the wind source.

- Bridging Power: This is considered as an emergency storage that provides energy ranging from seconds to a few minutes when a change in the source of generation is being carried out. In regard to wind power generation, this is not a direct application, since it is not a bridge power supply, but a long term source. Therefore, the storage system does not improve the performance of the wind farm, but it has a positive effect on the ordinary operation of the network when switching from one source to another.

- Energy Management: The principle of this application is basically storing energy when generation surpluses occur and thus providing when consumption exceeds generation. This technique allows decoupling consumption and generation, which is known as load levelling. The energy is then released for a longer period of time (from minutes to hours). This option is also the most profitable alternative, since the energy can be stored during off-peak hours, and then released during peak hours, when the cost of electricity is 
higher, obtaining therefore economical benefits. The future use of storage systems for this application is regarded as an interesting solution for the random long-term variability of wind. By operating under these conditions, very few energy is wasted. When wind generation exceeds electric consumption, energy is stored, being available afterwards for a long-term supply. This makes this option ideal for isolated wind generation and also enhances the advantages of network-connected wind power.

Each of these alternatives requires specific characteristics of the storage device utilised. To operate under power quality control, a huge response of power during a short period of time is needed. Meanwhile, for the energy management, the device must be able to be energetically available for hours. Therefore, it is important to compare and contrast their main properties, in order to decide which is the most suitable for a particular application.

Table I [4] shows the relative importance of the different characteristics according to a power or energy application.

Table I. - Energy and Power Requirements [4]

\begin{tabular}{|r|c|c|}
\multicolumn{1}{c|}{} & POWER & ENERGY \\
\hline Large storage capacity & - & + \\
\hline Large power capacity & - & + \\
\hline Power density & + & + \\
\hline Energy density & + & - \\
\hline Power gradient & ++ & - \\
\hline Cycle life time & + & + \\
\hline Calendar life time & + & + \\
\hline Access time & ++ & - \\
\hline Low self-discharging & - & + \\
\hline \multirow{2}{*}{++ very important } & + important & - less important
\end{tabular}

As it can be seen, for power applications it is essential for the device to have a short access time and a high power gradient; while for energy applications the large storage capacity, low self discharging and long life time are the most remarkable requirements.

\section{Comparison}

A comparison among the different storage systems must be done in order to decide the proper device for each use. As seen in the previous section, devices can be roughly classified according to their suitability for energy or power application. However, a deeper study of their characteristics is required.

A substantial number of parameters are analysed when comparing different storage systems. Some of these are efficiency, costs, autonomy, environmental impact or reliability among others. This work focuses on the most remarkable technical characteristics when a decision has to be made. Therefore, the devices will be compared in terms of their storage capacity, available power, discharge time, lifespan, access time and self-discharging rate. All these values are shown in Table II for the chosen devices. In case there are no noticeable technical differences between different options, costs will also be considered when comparing the alternatives.

As seen in Table II, batteries have higher storage capacity than the rest of devices, which is a crucial parameter for a proper behaviour in energy applications. Likewise, the hydrogen technology, using fuel cells to produce electricity from stored hydrogen, provides considerable amounts of electricity during long periods. Besides, the discharge time of all these devices covers from seconds to days, being most of them able to operate for hours.

Among batteries it is possible to find a large variety of devices. All of them have different features and are currently in dissimilar stages of development. Some batteries are optimised for power applications, others for energy, but in very few cases are they optimised for both. Lead-acid and Li-ion stand out among the electrochemical group. The former has been widely utilised in several usages. It is the most mature technology and has proved an adequate behaviour either under energy or power requirements. Its experienced capabilities, as well as its low self-discharge rates, support its massive use for energy supply, whereas its applications are limited by the relatively short life time it presents. The latter is able to substitute the lead-acid technology in most of the cases. Furthermore, Li-ion batteries present a higher energy density in both weight and volume, with efficiency close to $100 \%$. These characteristics make Li-ion batteries to be the most popular choice for portable devices. However, the excessively high costs (between 900\$/kWh and $4,000 \$ / \mathrm{kWh}$-depending on the rated power-, in contrast to a maximum of $1,000 \$ / \mathrm{kWh}$ for lead-acid [5]), and its electrical fragility -i.e. serious damages due to deep discharge- prevent its use as a large-scale energy storage system.

The different types of nickel-based batteries have a higher energy density and a longer life-time than the lead-acid technology. Nevertheless, they have not been introduced to a commercial use mainly because of their higher costs and self-discharge rates, and their lower electrical efficiency. Eventually, metal-air batteries present a major drawback with the fact that it is fairly difficult to recharge them effectively, what hampers its developments as an adequate alternative to traditional systems.

Redox flow and sodium sulphur batteries appear as the most interesting alternatives in the near future. These types are currently in use in several locations. Some authors consider the $\mathrm{NaS}$ batteries the best option when storing wind energy production. They are able to supply an amount of energy during a considerable long time, or otherwise, a power pulse in a short instant, maintaining a high efficiency. They also present a short access time, which helps reducing short-term wind power fluctuations. However, these batteries require a frequent heat input to maintain electrolytes in a molten state. As a result, they are being used with positive results in power quality large-scale applications, but they are still a growing technology. 
Table II - Storage technologies

\begin{tabular}{|c|c|c|c|c|c|c|c|}
\hline $\begin{array}{c}\text { Storage Capacity } \\
{[\mathbf{k W h}]}\end{array}$ & $\begin{array}{c}\text { Electro- } \\
\text { chemical }\end{array}$ & Redox Flow & NaS & Flywheel & $\begin{array}{c}\text { Super- } \\
\text { capacitor }\end{array}$ & SMES & Fuel Cell \\
\hline $\begin{array}{c}\text { Available Power } \\
{[\mathbf{k W ]}}\end{array}$ & $0.5-10^{3}$ & $500-10^{5}$ & up to $2 \cdot 10^{5}$ & $1-30$ & $0.1-0.6$ & $10-50$ & several $10^{3}$ \\
\hline Discharge Time & sec - hours & min - days & sec - hours & sec - min & seconds & sec - min & min - days \\
\hline $\begin{array}{c}\text { Lifespan } \\
\text { [years] }\end{array}$ & $5-15$ & 15 & 15 & 20 & 15 & 20 & 10 \\
\hline $\begin{array}{c}\text { Access Time } \\
{[\mathbf{m s}]}\end{array}$ & 20 & 1 & $5-15$ & $2-5$ & 0.5 & $0.5-5$ & 20 \\
\hline Self-discharging & $\begin{array}{c}2 \%-5 \% \text { per } \\
\text { month }\end{array}$ & None & None & $\begin{array}{c}20 \% \text { per } \\
\text { hour }\end{array}$ & $\begin{array}{c}14 \% \text { per } \\
\text { month }\end{array}$ & $\begin{array}{c}\text { Cooling } \\
\text { power }\end{array}$ & $\begin{array}{c}3 \% \text { per day } \\
\text { (tank) }\end{array}$ \\
\hline
\end{tabular}

So are the flow batteries. They stand out for their high energy and power capacity. Besides, their principal advantage is the ability to decouple these properties during the design stage. In addition, the lack of selfdischarge, and the absence of deep discharge damaging, together with a long discharge time, make this technology suitable for long duration storages. Nevertheless, its extensive use faces important investment and running (due to the need for pumping the liquid electrolytes) costs that hinders the commercial development of this technology.

Energy applications based on hydrogen generation are under constant research. This system is not recommended for power applications, since impurity in hydrogen may appear when operating under constant switching, thus reducing life expectancy. Electricity generation via fuel cells produces no polluting wastes. Besides, its long discharge time permits an energy support for a few days, being the leading technology in this respect. Even though fuel cells are still in the beginning of their commercial stage, they are already available in low power. Electrolyzer and hydrogen storage combined with fuel cell generation provide a very low efficiency system, approaching $40 \%$, which is one of the main drawbacks. Investment costs are excessively high, as well. These reasons dissuade from a wider use of hydrogen technology for massive energy supply. However, fuel cells can work properly in decentralised production, midpower utilities and isolated areas, as stated in [6].

On the other hand, for power applications different devices -such as supercapacitors, SMES or flywheelsare preferred. All of them reach sufficient power rates, and their storage capacity is not high enough for a long duration use. In fact, they may cover a maximum supply of only a few minutes. Besides, their considerable selfdischarging rates do not contribute to the energy management applications. It is difficult to say whether a certain device or another is better than the others, since all present a proper performance in different situations. This is particularly remarkable in the field of wind energy generation, since these three devices are specially recommended to mitigate the effects of wind speed random fluctuation. Therefore, the main characteristics of supercapacitors, SMES and flywheels will be stated, in order to decide the most suitable profile for each.

With regard to Table II, supercapacitors present the lowest storage capacity and power response. Nevertheless, they have an exceptional power density, up to $10,000 \mathrm{~W} / \mathrm{kg}$ [1], which can be released and restored within a few seconds, with energy efficiency over $95 \%$. These inherent properties suggest the supercapacitors as a good option for peak power supply, providing power back up to sudden power demands [1]. However, it is a still young technology, which bears greatly high costs, most of the times unacceptable for certain applications.

SMES systems show a fast response time, in which they are able to provide a huge power pulse with a good overall efficiency. Due to their configuration, life time is not affected by frequent and deep discharge, as opposed to conventional batteries. Hence, they are particularly interesting when complete charge-discharge cycles are required on a continuous basis [6]. Moreover, since they allow a few minutes long operation, small-scale energy management is feasible, as well as power quality control with intermittent sources. A major drawback is the cryogenic temperatures needed for cooling the system, which consume great amounts of energy, increasing the operational costs of these storage systems. Besides, huge magnetic fields are necessary, producing stability problems and requiring special infrastructure conditions. These shortcomings leave the SMES system far behind from other more developed technologies.

Flywheels are currently the leading technology in the field of power quality control, since it is supported by its successful results in a number of industrial applications. Its short access time, high power rating and excellent cycling capacity (long lifespan) promote its use as power back up for decentralised generation and renewable, uneven energy production. However, the remarkable selfdischarge rate they present hinders a long-term energy supply. Besides, while the capital cost for high-power flywheels is lower than for SMES devices or supercapacitors, it still needs to be reduced to be able to compete with lead-acid batteries or other more mature systems. 


\section{Conclusions}

Once the different technologies have been analysed and compared, it is necessary to state the conclusions drawn from the study.

First of all, it is essential to highlight the fact that energy storage has proved to be economically affordable and technically feasible in most of the cases [6]. Despite the costs related to storage, its importance will increase as further development of the different technologies is carried out.

Some of the consulted references conclude that lead-acid batteries are nowadays the most widely utilised storage system, either for power or energy applications. Although other technologies have accomplished better performances in specific applications, the large experience acquired by lead-acid is still a key factor when comparing among different options. Moreover, a massive use allows cost reduction, thus benefiting its leadership.

For energy management applications, lead-acid batteries are the preferred option. They present acceptable performance at relatively low cost. However, a great effort is being made in order to find a more suitable storage system. Redox flow and sodium-sulphur batteries, as well as hydrogen technology, seem to be the future alternatives for these applications. For all of them, investment in R\&D must be done. The first two need to grow and reach a larger commercial relevance. Improving current techniques will reduce operational costs, thus providing a more economically competitive scenario, in which these batteries will replace the less efficient already existing ones. In the case of hydrogen, a strong increase in efficiency is required. Its slightly developed technology leads to a poor efficiency and an expensive system. New storage technologies, which will improve energy and power density, are under research. Coupling higher energy density with more efficient fuel cells will make the hydrogen technology the least environmentally damaging option, with fairly high electricity generation possibilities. Besides, it can be used in mobile applications such as hydrogen vehicles. Nevertheless, all these advances ought to produce a drastic reduction in cost, in order for this system to be commercially available.

Lead-acid batteries are widely used in power quality applications as well. However, flywheels, SMES systems and supercapacitors will substitute this technology as soon as they become more affordable. All these three options present excellent power responses as well as a very short access time. Moreover, they are more reliable and long durable than lead-acid. Among the three, flywheels and supercapacitors are currently better positioned. The former has a longer experience, showing satisfactory results in a number of applications, while supercapacitors are able to reach extraordinary power density with fast and efficient charge-discharge cycles.
On the other hand, SMES systems have important difficulties due to the use of large magnetic fields. It is necessary to improve the power electronics in these systems to better solve the stability problems produced by magnetism. Since special infrastructures are required -i.e. underground locations-, it is needed to find more economically attractive options for this technology to be used on a commercial basis.

Since for bridging power applications a mixture of all the previously stated characteristics is desirable, flow batteries and SMES or flywheels are feasible. They present good power and energy capabilities, together with a short access time and a discharge time that ranges from seconds to several hours if working coupled.

It is important to take into account the benefits obtained when coupling different storage systems. Installed in a wind farm, an energy storage system with such a wide range of possibilities will optimise electricity production to a maximum. In addition, energy costs will decrease thus making wind power a more competitive source.

In conclusion, the development of new and better energy storage systems promises a growing scenario for wind power generation. The use of advanced technologies (alone or coupled) optimises electric production, hence reducing generation costs. Besides, a higher penetration of renewable and clean sources means a subsequent reduction of fossil fuels power stations. Therefore, it is crucial to invest in $R \& D$ for the different systems presented in this study. At the same time, further fieldwork is necessary in order to increase the knowledge of their performance under real operating conditions, and gain in experience. Work must focus on the enhancement of the overall capabilities of these devices, since they have proved to be the most suitable alternatives for the different working features considered.

\section{References}

[1] I. Hadjipaschalis, A. Poullikkas and V. Efthimiou, "Overview of current and future energy storage technologies for electric power applications", Renewable and Sustainable Energy Reviews 13 (2009), pp. 1513-1522.

[2] K. C. Divya and J. Østergaard, "Battery energy storage technology for power systems - An overview", Electric Power System Research 79 (2009), pp. 511-520.

[3] S. A. Sherif, F. Barbir and T. N. Veziroglu, "Wind energy and the hydrogen economy - Review of the technology", Solar Energy 78 (2005), pp. 647-660.

[4] E. Spahić, G. Balzer, B. Hellmich and W. Münch, "Wind energy storages - Possibilities", PowerTech 2007, pp. 615620

[5] http://www.electricitystorage.org

[6] H. Ibrahim, A. Ilinca and J. Perron, "Energy storage systems - Characteristics and comparisons", Renewable and Sustainable Energy Reviews 12 (2008), pp. 1221-1250. 